\title{
Protective Effect of Probiotics Strains in Ruminants
}

\author{
Everlon Cid Rigobelo and Fernando Antonio de Ávila
}

Additional information is available at the end of the chapter

http://dx.doi.org/10.5772/50054

\section{Introduction}

In last 15 years the use of probiotics strains in animal production has been increased. These probiotics strains can modulate the balance and activities of the gastrointestinal microbiota in which are responsible to gut homeostasis. The intake of probiotics supplemented in ration and provided to the animals, can strongly affect the structure and activities of the gut microbial communities leading to promoting health and improving the performance in livestock, when it is impaired by numerous factors, such as dietary and management constraints. The understanding of the digestive ecosystems in terms of microbial composition and functional diversity is fundamental to modulate the gastrointestinal tract (GIT) of domestic animals providing to them the possibility to maintain the homeostasis of these complex microbial communities, which can be composed of bacteria, protozoa, fungi, archaea, and viruses, thus promoting a reduction of the incidence of diseases. Therefore considerable researchs during 30 years are characterizing the domestic animals 'GIT. The welfare, health and feed efficiency of the animals can be affected by different factors, many of them, environmental factors. Among these factors, feeding practices, composition of animal diets, farms management and productivity constraints can influence the microbial balance in GIT, whose role is fundamental to gut homeostasis and its reduction consequently can affect efficiency digestive When occurs the reduction of microbial in GIT, some reactions as digestion and fermentation of plant polymers are impaired, since the action of the microbiota on gut is strongly related with the realization these reactions, and the animals also are impaired by the fact these polymers to be of particular importance to the herbivorous (Chaucheyras-Durand and Durand, 2010).

\section{Use of antibiotics}

The problem caused by indiscriminate use of antibiotic as growth promoter in feed to livestock is that this practice has been associated with emergence of resistance to antibiotics 
in zoonotic bacteria. The use of growth promoter in feed to livestock has been done since 1940 because this practice is correlated with higher health status and improves at performance of animals in terms of feed conversion. The use of antibiotics at animal has had a profound impact on animal health and welfare.

The problems found by this practice require the development of alternative intervention strategies for zoonotic livestock pathogens. Some these strategies could be vaccines in diarrhea in neonates and post weaning animals, limited access to livestock, control of vermin, modifying air flow, high level disinfection regimes, acidification of feed and the supply of probiotic into animals supplemented in ration by example are efficient management to reduce the occurrence of pathogen at the animal production.

\section{Use of probiotics strains}

All additives used in animal feed, including yeasts and bacteria, are strictly regulated within the EU legislative framework. Until May 2003, the risk assessment of animal feed additives for use in European was the responsibility of the Scientific Commitee of Animal Nutrition (SCAN) (Anadon et al., 2006). After this date, the European Food Safety Authority (EFSA) took over the functions of SCAN. While EFSA provide expert scientific advice to the European Commission the approval and risk management of a probiotic product is responsibility of the EC and its constituent member's states. For use of microorganism in United States as a feed additive is necessary before the product to be outgoing to approval by the Food and Drug Administration (FDA).

The requirements for a novel probiotic product required by EU regulations on animal feed additives are the identification and characterization to species level, and the efficacy data must be provided in support of any claims made for the product. Some characteristics are requested to product such as no adverse effects on the health of performance, the product must be safe for the operator, have no adverse effects upon exposure and also the product must not pose a risk to the safety of the end-consumer (SCAN, 2001).

\section{Use of probiotics to control gastrointestinal diseases in livestock}

The intensive production farmed livestock together with the veto of the use of antimicrobial feed supplements in the EU, this situation has increased the risk of contracting gastrointestinal diseases if prophylactic antimicrobial feed supplements are not utilized. The removal of growth promoters has led to a significant increase in the incidence of diseases and also with significant increases in feed costs, the reduced feed weight conversion.

\section{Use of probiotics in animals}

Although the mechanisms involved have not been fully elucidated a reduction in pathogen carriage and subsequent clinical disease is one possible mechanisms responsible by reduction of occurrence of disease when the growth promoter is utilized in livestock. After 
this prohibition many problems arisen and also the need of use of alternatives to resolve this situation. One of these alternatives is the use of probiotics as feed supplement or functional food which may be used for prophylaxis in animals and humans. There are numerous probiotics products commercially available for livestock. Currently commercial livestock probiotic can be separated into two categories, being these, competitive exclusion that are defined and those that are undefined.

\section{Use of probiotic in ruminants}

In ruminants that have four stomachs being them rumen, omasum, reticle and abomasums when these animals born they have the abomasums extremely big. This situation occurs because the type of food is liquids as milk. Usually the animal becomes ruminants when he from the third or fourth month of age. This development is due the installation of microbiota ruminal in gut and also by distention of organ due the fiber intake. The bacteria from rumen and bowel are acquired through the contact of cattle with the cow or other animal and also by grass intake.

The rumen is as fermentation chamber and it has approximately for $50-85 \%$ by use of dry matter from food. The saliva is mixed with food and has a control upon $\mathrm{pH}$ of rumen and the papillae existing in inner wall of the rumen increasing the absorption area.

The amount of bacteria from rumen is the approximately $10^{11} \mathrm{CFU} / \mathrm{g}$ of counts rumen, the fungi is the $10^{3} \mathrm{CFU} / \mathrm{g}$ and the protozoa is $10^{5} \mathrm{cell} / \mathrm{g}$. There are most of 60 species of bacteria that grow into rumen microbita and this environment has $\mathrm{CO} 2, \mathrm{CH} 4$ and $\mathrm{N} 2$ stomach gas maintaining the $\mathrm{pH}$ value among $6-6.5$. The temperature within the rumen is $39^{\circ} \mathrm{C}$ and the bacteria type living can be characterized according to theirs functions such as cellulolytic, proteolytic, amylolytic.

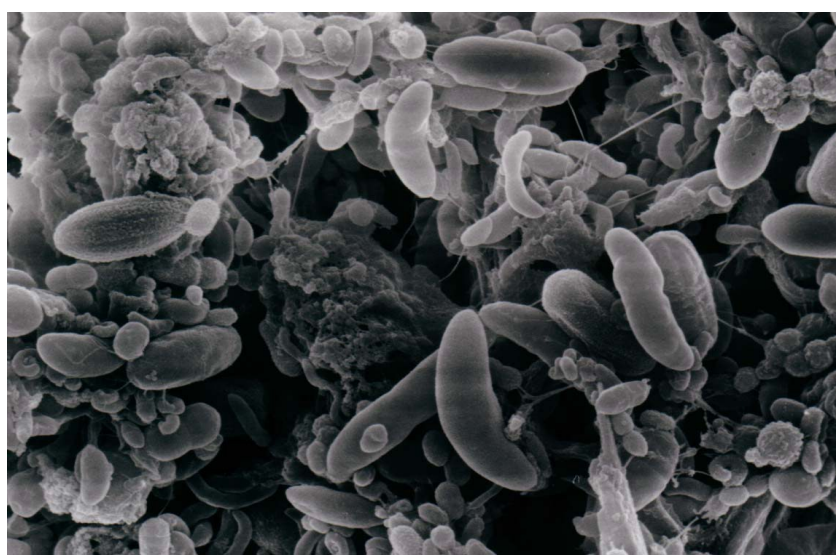

Picture 1. Picture took from Antibiotics and chemotherapeutic and probiotics Avila et al Funep Publisher Brazil 83p.

The proteins and fibrous foods in rumen are converted at ammonia, organic acids and amino acids by microorganism's action. As the majority of amino acids are synthesized of 
rumen the animals need to be supplied with essential amino acids from ration or injectable. The main factors of stress feed that leaving to a decreasing of ruminal microbiota are dry grasslands, pastures in budding and seasonal changes. The decreasing of ruminal microbiota can be caused by antibiotics use and also environment changes as occur at auctions, expositions and pre-slaughter. The use of rumen bacteria into ruminants promotes the growth into gut before the establishment of pathogen in these animals causing the prevention of diarrhea occurrence. This situation decreases the weaning time and maintains the balance of rumen microbiota increasing the production of enzymes as cellulase, amylase, urease, protease consequently increasing improving the use fibrous foods. Others benefits to use of probiotics in ruminants are promotes the increasing of weight gain, increasing the milk production and decreasing of diarrhea period.

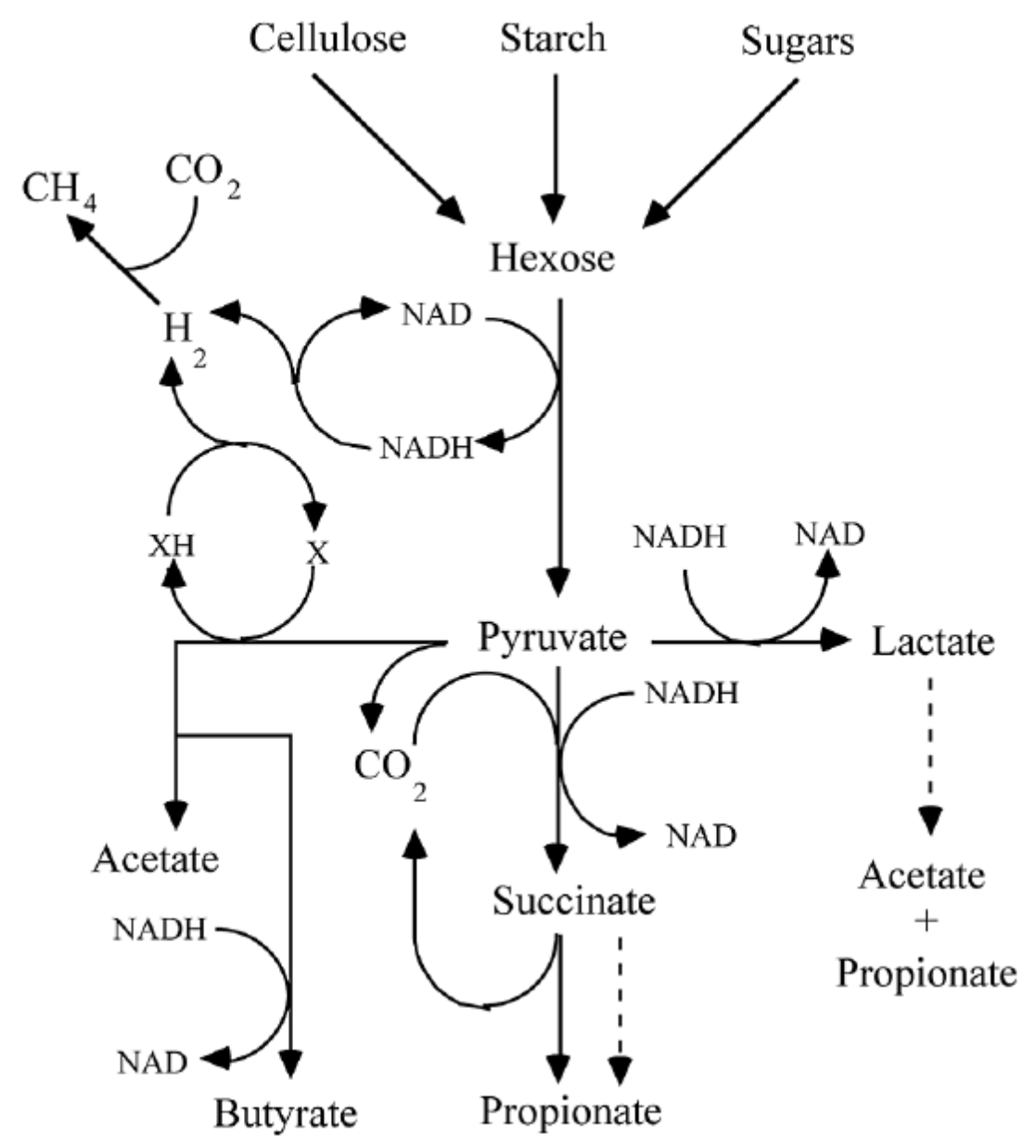

Picture 2. Picture took from Antibiotics and chemotherapeutic and probiotics Avila et al Funep Publisher Brazil 83p. 
The advantages of the use of probiotics in livestock are the period of adaptation of animal is not necessary, doesn't hinder the management on the farm because it can be supplement to ration or mineral salt, and as probiotic is the natural product does not necessary the disposal of milk and also this product can be used during the slaughter of animals as cattle, sheep and buffaloes. According with FERREIRA, (2003) the probiotics microorganisms most used belong to the group of lactic bacteria as Aerococcus, Atopobium, Bifidobacteirum, Brochothrix, Carnobacterium, Enterococcus, Lactobacillus, Weissella. The lactic bacteria are positive Gram, anaerobic, negative catalase, presenting of cocos and bacillus way. The probiotics can counts ruminal bacterias as Ruminobacter and Succinovibrio with specifics characteristics that are used in supplementation of ruminants.

Some authors have been showed that some probiotics strains have seen resistant to the antibiotics effects and therefore these strains could be used together the administration of antibiotics in animals. The yeasts are unicellular microorganisms with capacity of survive in several mediums have a great spectrum of $\mathrm{pH}$ and many mediums can be saline or without oxygen. The Saccharomyces boulardii has been largely tested in human's trials (PENNA et al., 2000). And the Saccharomyces cerevisiae in animals showed promising results.

The Lactobacillus is constituted by cells that vary long and thin to short and curves with 1.5$6.0 \mu \mathrm{m}$ length and 0.6-0.9 width. The ideal temperature to growth is $45^{\circ} \mathrm{C}$ and grows in $\mathrm{pH}$ 5.5-6.0. The Lactobacillus species known at moment is 56 and the most used as additive are L. acidophilus, L. rhamnnosis and L. casei.

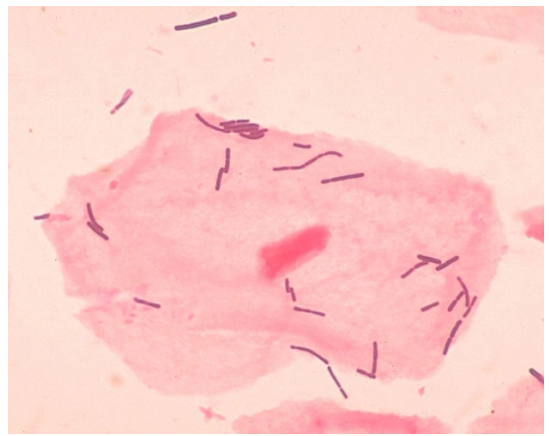

Picture 3. Picture took from Antibiotics and chemotherapeutic and probiotics Avila et al Funep Publisher Brazil 83p.

The genus Bifidobacterium includes 30 species. Many of these 10 are form humans dental caries, vagina and feces, 17 are from animal origin 2 are from wastewater and 1 of fermented milk. These bacteria present optimal growth among $37^{\circ} \mathrm{C}$ and $41^{\circ} \mathrm{C}$ and minimal growth among $25^{\circ} \mathrm{C}$ and $28^{\circ} \mathrm{C}$ at $\mathrm{pH}$ 6-7. The Bifidobacterium Bifidobacterium animallis, Bifidobacterium lactis, Bifidobacterium longum species have probiotics characteristics also have capabilities to ferment complex carbon.

Some species of Bacillus subtilis, Bacillus licheniformis and Bacillus cereus are bacteria positive Gram in rods form. The Bacillus are the only that form spores allowing that these strains to be used in adverse conditions mainly in high temperature. 


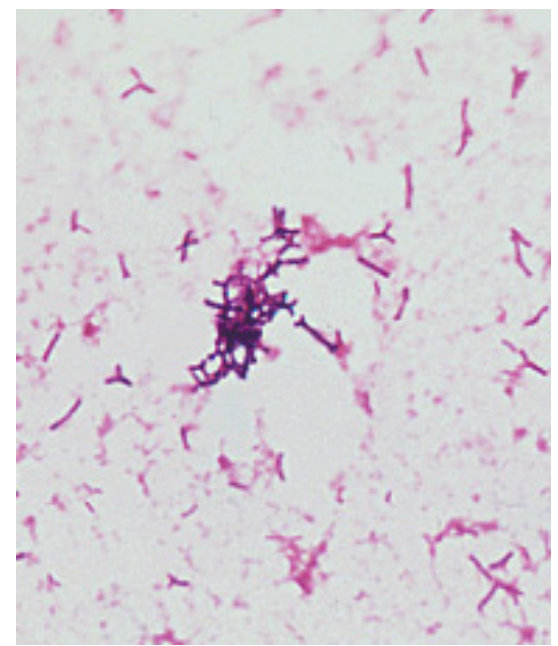

Picture 4. Picture took from Antibiotics and chemotherapeutic and probiotics Avila et al Funep Publisher Brazil 83p.

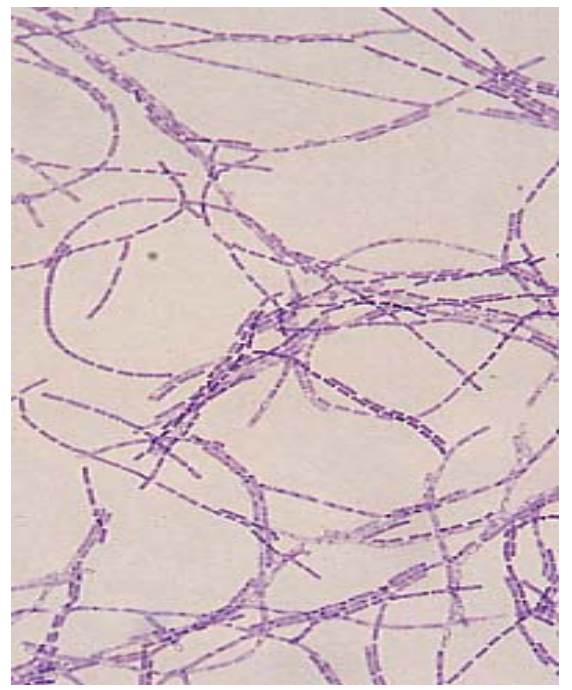

Picture 5. Picture took from Antibiotics and chemotherapeutic and probiotics Avila et al Funep Publisher Brazil 83p.

Enterococcus faecium is the microorganism belonged to the Enterococcus genus belonged to the Lancifield D group. This morphology identification requests the use of coloration by Gram and also catalase test in blade. These bacteria are positive Gram and present the characteristic form of streptococcus (chain cocos), negative catalase and no spore and faculty anaerobic. Through the chemical analysis the strain ferment the lactose, arabinose, mannitol, no ferment the sorbitol. This strain growth into MacConkey medium containing $6.5 \%$ of $\mathrm{NaCl}$. 


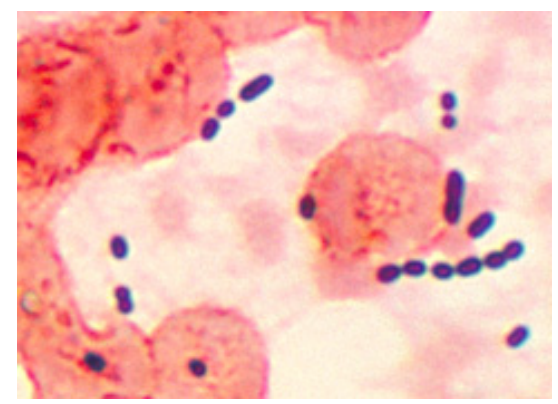

Picture 6. Picture took from Antibiotics and chemotherapeutic and probiotics Avila et al Funep Publisher Brazil 83p.

Ruminobacter amylophilum is the microorganism belonged to the Ruminobacter genus to its morphology identification is necessary to use Gram coloration this genus present as rods negative Gram. They have motility and no spores. This ferments the cornflour, maltose and liquefy gelatin. They synthesize lactic acid and $\mathrm{CO} 2$ from formic acid.

\section{Others benefits and action mode of probiotics strains}

\subsection{Immune modulation}

The maturation of the humoral immune mechanisms can be conducted by microbial colonization, this events can promote the c circulation of the $\operatorname{IgA}$ and $\operatorname{IgM}$ secreting cells. The other important factor that can be affected by microbial colonization on the gut of different animals particularly the ruminants are the balance of the different $T$ helper subsets. The memory B and T cells migrate to effectors sites in consequence these events.

Other mechanisms to immune modulation are followed by active proliferation local induction of certain cytokines and production of secretion antibodies as IgA. When the host is exposure to the antigen, immune cells respond releasing cytokines from host direct the subsequent immune responses. The low-dose tolerance immunity TGF-B associated in via local cytokine is the man mechanisms which the gut associated lymphoid tissue maintains homeostasis. Some lactic acid bacteria can induces the production of proinflammatory cytokines, tumor necrosis factors alpha and interleukin-6 from human peripheral blood mononuclear cells. A strain of Lactobacillus casei can inhibit the growth of pathogenic strains as Pseudomonas aeruginosa and Listeria monocytogenes leading to an increase in the level of macrophages. Others strains as Lactobacillus acidophilus and Bifidobacterium bifidum could inhance non-specific immunity and concluded that specific lactic acid bacteria could play a role in specific age groups, specific neonates or the elderly. The results can be observed when two groups of animals are compared itself in relation with their age. Usually the positive effect against the colonization by pathogenic bacteria upon the gut occurs most efficiently in neonates than oldest.

Some studies showed a significant increase in IgA immune response. In others, on children with mild to moderate stable Crohn's Diseases, administration with strain GG improved the gut barrier function and clinical status after six months of therapy. 


\section{Antitumor activity}

Some probiotic strains could decrease some enzymes synthesized by many microorganisms may convert procarcinogens into carcinogens and cause colon cancer, some of them azoreductase, $\beta$ - glucuronidase and nitroreductase. Lactobacillus acidophilus could decrease nitroreductase, azoreductase and $\beta$ glucuronidase activities in carnivorous animals. Another strain as Lactobacillus rhamnosus could bacterial $\beta$-glucuronidase activity in the large intestine.

Lactobacillus and Lactobacillus bulgaricus suppressed Ehrlich ascitis tumor or Sarcoma 180 in mice. Tumor suppression in associated with intact viable cells, intact dead cells and cell wall fragments or Lactobacilli and Bifidobacteria. When Lactobaacillus casei was provided into rats it had effective prevention against the recurrence of superficial bladder cancer.

Nitites used in food processing are converted to carcinogenic nitrosamines in the gastrointestinal tract in several people. Cellular uptake of nitites by Lactobacillus and Bifidobacteria has been shown in vivo. Also, Lactobacillus has been shown as a great reducer of bile salts. They are implicated in the initiation of colon carcinogens. These strains have been biotransformed of primary to secondary bile salts, this way, there are reduction the possible initiation of cancer. Other authors have been suggested that the decrease of intestinal $\mathrm{pH}$, through metabolic activities of Lactobacillus acid bacteria, could inhibit the growth of putrefactive bacteria, can prevent large bowel cancer.

Many probiotics strains have a positive effect against mould growth and aflatoxin production. These aflatoxins are associated to cause cancer. Thus the reduction of these moulds decrease the occurrence of cancer caused by this mould.

\section{Reduction of cholesterol}

Some studies have showed the effect of fermented milk or milk containing probiotic strains producing lactic acid on serum cholesterol levels. These studies reported that a strain of Streptococcus thermophilus and Lactobacillus acidophilus reduced cholesterol levels in rats. Milk fermented with lactic acid bacteria and Streptococcus cerevisae led to lower serum cholesterol than control group, also phospholipids and bile acids in the fecal samples from mice were lower. When a trial was using rats inoculated with E. faecium, they presented a lower cholesterol levels. The same findings were observed in pigs that have been fed a high cholesterol diet.

Another results also, showed that the serum lipoprotein levels of 334 individuals remained unchanged when they were treated with Lactobacillus acidophilus and L. delbrueckii subsp bulgaricus and E. faecium administered over six weeks to adults and it resulted in a initial increase in total cholesterol and LDL followed by a sharp decrease two weeks after termination of treatment. The decrease corresponded with an increase in the reduction of iodonitrotetrazolium and superoxide production by peripheral neutrophils and an elevated production of IgG. Several studies don't explain because there was the reduction in 
cholesterol levels and suggest that the reduction of cholesterol is not due to assimilation or to a direct interaction between the bacteria and cholesterol. This effect is due to the coprecipitation of cholesterol with deconjugated bile salts at $\mathrm{pH}$ value below 6.0. This would not explain the reduction of cholesterol in vivo as the $\mathrm{pH}$ of the bower gastrointestinal is neutral to alkaline. Probably there is a physical association between cholesterol and the cell surface.

\section{Decreasing of lactose intolerance}

Some descents from Asia and Africa usually are stricken by lack the intestinal mucosal enzyme $\beta$-galactosidase and therefore suffer from reduction in lactase activity. This situation can occur many times after an infection caused rotavirus gastroenteritis. There are much lactic bacteria which are capable to synthesize the enzyme $\beta$-galactosidase. Many of them as the bacteria Streptococcus salivarius subps thermophilus and Lactobacillus delbrueckii subps bulgaricus. The levels of enzyme produced by these bacteria are high and many products treated with this enzyme presented a low concentration of lactose. These species are sensitive to bile salts. These substances can lead to release of high levels of $\beta$-galactosidase in the gastrointestinal tract. Lactose from fermented milk containing the probiotic Lactobacillus acidophilus were better absorbed by many people with lower $\beta$-galactosidase activity. All symptoms from lactose intolerance were decreased.

\section{Stool transit}

The diarrhea occurrences in neonate are the main cause of death. This disorder affects animals of many species and also the human among them the children. Lactobacillus GG had a high decreasing in severity of acute watery diarrhea in young children. Patients treated on erythromycin reacted decreasing the period of diarrhea when they received Lactobacillus GG.

The symptoms caused by slow stool transit are diarrhea, stomach pain, abdominal pain and nausea. All symptoms were recovery quickly when the patients received Lactobacillus GG. Indeed one of the most severe diarrhea is that caused by Clostridum difficile. Usually people stricken by this disease recently passed by treatment with antibiotics. The supply of Lactobacillus rhamnosus improved the symptoms of intestinal disorders.

Patients who consumed milk fermented by the strain experienced less diarrhea than those that don't received. Many of them were patients that were being treated with pelvic radiotherapy. The effect of different LAB $n$ different types of diarrhea has been showed in many studies. Yet are needed others studies to determine which mechanisms the LAB use to relieve diarrhea.

From now on this chapter will present some findings from some trials that were performed with the aim of verifying the protective effect of a probiotic mix that was kindly donated by IMEVE Biotecnology located in Jaboticabal São Paulo State against the colonization caused by STEC in sheep. 
Abstract: Shiga toxin-producing Escherichia coli (STEC) strains are food-borne pathogens that cause human diseases, and ruminants are usually important reservoirs of STEC. The first step of enteric infection is colonization of the host's gut mucosal surface by pathogenic strains of bacteria. Probiotic bacteria can decrease the severity of infection by competing for receptors and nutrients and by synthesizing an acid that creates an unfavorable environment for the growth of several bacterial species. The aim of this study was to determine whether the inoculation of sheep with a mixture containing $5 \times 10^{8}$ (CFU) of Lactobacillus acidophilus, Lactobacillus helveticus, Lactobacillus bulgaricus, Lactobacillus lactis, Streptococcus thermophilus and Enterococcus faecium per animal decreases the shedding at animals previously inoculated with STEC nonO157. Sheep that received oral inoculums containing $2 \times 10^{9}$ viable bacteria of STEC carriers of st $x 1$, st $x 2$ and eae genes were compared with others groups that did not receive inoculums. When probiotic was inoculated together with the STEC non-O157, the numbers of these same bacteria in a fecal sample were lower than the group did not receive. It occurred during the $3^{\text {th }}, 5^{\text {th }}, 6^{\text {th }}$ and $7^{\text {th }}$ weeks postinoculation. Thus, we conclude that this mixture likely presented a potential protective effect in reducing colonization by STEC non-O157 and can be used as an alternative method to decreases STEC non-157 infection in sheep, thereby reducing transmission to humans.

\section{STEC diseases}

Healthy cattle, sheep and other ruminants can be reservoirs of Shiga-toxin-producing Escherichia coli (STEC) strains. STEC have been associated with human diseases such as hemorrhagic colitis and hemolytic uremic syndrome (Hussein 2007; Ramamurthy 2008). These bacteria can be transmitted from person to person (Belongia et al., 1993), but most outbreaks have been associated with the consumption contaminated beef products or a variety of other foods. Before colonization by STEC, it may be possible to determine whether to use the colonization of ruminal mucosa by oral administration of probiotic bacteria as a strategy (Ávila et al., 2000).

Probiotics are live microorganisms that, when administered in the appropriate amount, will benefit the health of the host (Food and Agriculture Organization of the United Nations, 2003; Sanders, 2003). Microbial interference is common to all genera and decreases the severity of infection by mechanisms involving nutrient competition, generation of an unfavorable environment, and competition for attachment or adhesion sites (ChaucheryrasDurand and Durand, 2010). Probiotics bacteria can stimulate the immune system through innate cell surface pattern recognition receptors or via direct lymphoid cell activation. Practical applications for this action of probiotics based on this characteristic include their use in anti-tumor, anti-allergy and immunotherapy treatments, but there is also increasing evidence that some probiotics can sufficiently stimulate a protective immune response to enhance resistance to microbial pathogens (Cross, 2002).

The benefits caused for use of probiotics strains in ruminants are known, however there are few information about the use of probiotics strains to reduction of shedding of STEC nonO157 in sheep. 
This study verified the protective effect of probiotic treatment against the colonization of STEC non-O157 in sheep measured the number of STEC recovered from fecal simple.

\section{Materials and methods}

\subsection{Animals and experimental locations}

The study was performed with 20 sheep of Santa Ines race in the fattening stage, female previously screened by not be carrying of STEC non-O157 strains distributed in four groups with five animals each that were confined at a property located in São Paulo State. The experiment was made January to March 2012. The sheep were selected based on closeness of body weight $(41 \pm 2) \mathrm{kg}$ and age (9-12) months. Then, all animals were ear-tagged and drenched with Ivomec (MSD- Agvet Merck) for internal parasite control at the rate of $2 \mathrm{cc} / 46 \mathrm{~kg}$ body weight. During three weeks pre-experimental adaptation period, were offered for all groups of sheep a diet of identical composition ad libitum consumption. Group I did not receive the probiotics strains or STEC non-O157 being the control group. Group II received an only oral dose of inoculums containing $2 \times 10^{9}$ viable cell of STEC non-O157 per animal. Group III received an only oral dose of inoculums containing $2 \times 10^{9}$ viable cell of STEC non-O157 per animal together with daily oral doses at concentration of $5 \times 10^{8} \mathrm{CFU}$ of Lactobacillus acidophilus, Lactobacillus helveticus, Lactobacillus bulgaricus, Lactobacillus lactis, Streptococcus thermophilus and Enterococcus faecium per animal lyophilized provided directly in the mouth of animals with help of a cannula of application throughout the experiment. The inoculums were provided with help of a cannula of application and were diluted at $40 \mathrm{~mL}$ of $0.9 \%$ saline solution. Group IV received the probiotics alone at the same number of cells viable and of the same way. During three weeks before of start of experiment always in same hour in the morning were collected feces samples directly of rectum of these animals. The samples were cultured in plate on MacConkey agar then the colonies that grew had their DNA extracted as described by Wani et al. (2003) to verify the absence of STEC non-O157 and Salmonella. After the third week the groups of animals were inoculated and monitored by seven weeks with weekly collections of their feces. All animals of present study were not carrying STEC nonO157 before inoculation and were kept in bays separated to avoid cross contamination throughout the experiment in an environmentally controlled building. Each pen had a concrete floor with individual drain, a feeding box and water through and was cleaned once a day and the fecal material deposited was transported to other place where it was composted.

This study was conducted in accordance with the ethical guidelines for investigations involving laboratory animals and was approved by the Ethics in Animal Research Committee (EARC) of UNESP-Univi Estadual Paulista and no adverse effects were observed in the animals receiving the E. coli (STEC) and probiotics during the experiment.

\section{Probiotic}

The probiotics bacteria used were Bacillus cereus, Lactobacillus acidophilus and Enterococcus faecium all strains in amount of $3 \times 10^{8}$ (CFU). These strains were isolated from sheep rumina and intestinal tracts following the recommendations of Hungate (1975) and Wolf et al. 
(1975). These bacteria have the following features: they are nonpathogenic, enzymeproducing and resistant to lactic acid and low $\mathrm{pH}$. These strains were kindly donated by Imeve Medications Veterinary Industry responsible by all tests realized concerning the quality and conditions of use.

\section{STEC non-O157}

To verify the protective effect of probiotics strains reducing the shedding of STEC was used a STEC non-O157 strain isolated from healthy sheep and characterized as described by Possé et al., (2007). It was kindly donated by Laboratory of bacteriological from UNESP Jaboticabal.

\section{Samples}

For seven weeks, post-inoculation feces samples in same hour in the morning were collected from the sheep and transported to the laboratory, where DNA was extracted. Bacterial strains grown overnight in nutrient broth (Sigma) at $37^{\circ} \mathrm{C}$ were pelleted by centrifugation at $12,000 \mathrm{~g}$ for $1 \mathrm{~min}$, resuspended in $200 \mathrm{~m} \mathrm{~L}$ of sterile distilled water, and lysed by boiling for $10 \mathrm{~min}$. Lysates were centrifuged as described above, and $150 \mathrm{~m} \mathrm{~L}$ of the supernatants was used as DNA template for the PCR (Wani et al. , 2003). All isolates were subjected to PCR; stx1, stx2, and eae genes were detected using the primers and PCR conditions described by China et al. (1996). Control reference strains were E. coli EDL 933 (O157:H7, stx1, stx 2, eae) and E. coli K12 (negative control).

\section{STECs recuperated}

The values of STEC in each sample were determined of two different methods of counting. In both $1 \mathrm{~g}$ of each fecal sample was collected, cultured on MacConkey agar, then it was incubated at $37^{\circ} \mathrm{C}$ for $24 \mathrm{~h}$. In the first counting, all colonies grown displaying similar genome to STEC non-O157 strain previously inoculated orally were counted. In second counting were selected at least five colonies per sample grown and then separated in STEC non-O157 displaying pattern genome the others isolates from E. coli that did not display this specific DNA patterns.

\section{E. coli STEC fingerprint by pulsed-field gel electrophoresis (PFGE) of chromosomal DNA}

Genomic DNAs from STEC non-O157 isolates cultured from sheep were prepared as previously described by Barret et al., 1994. The agarose-embedded DNA was digested with $10 \mathrm{U}$ of $\mathrm{XbaI} /$ plug (Gibco BRL) at $37^{\circ} \mathrm{C}$ overnight. PFGE was performed in a CHEF-DR II unit (Bio-Rad Laboratories, Hercules, Calif.) using 1\% PFGE grade Tris Borate EDTA buffer gels. The DNA was electrophoresed for 20 hours at a constant voltage of $200 \mathrm{~V}(6 \mathrm{~V} / \mathrm{cm})$ pulse time of 5 to $50 \mathrm{~s}$, an electric field angle of $120^{\circ}$ and a temperature of $15^{\circ} \mathrm{C}$ before being stained 
with ethidium bromide. Resulting patterns were analyzed on a DNA Pro Scan, ProRFLP program (DNA Proscan, Inc. Nashville, Tenn), and the size of the DNA fragments was used as the criteria for categorizing distinct patterns.

\section{Results}

The animals received inoculums containing only one isolate of STEC non-O157 carriers of stx1, stx2 and eae genes. After three day post inoculations fecal samples were collected from these animals to make the re-isolating of the strains STEC non-O157 that had been previously inoculated into animals. All strains isolated from fecal samples had their DNA patterns compared with DNA pattern from STEC non-O-157 strain previously inoculated into animals and all those strains had the DNA similar to the strain previously inoculated were counted.

From strains isolated from fecal simples collected during the three weeks prior to inoculation of animals no STEC strain had the similar DNA to the DNA pattern from strains of STEC non-O157 previously inoculated into animals. The results showed that the STEC non-O157 strain previously inoculated into animals was the only strain recovered displaying this specific pattern of DNA. All strains isolated from fecal sample from animals from group I and IV also had no similar DNA patterns to the strain previously inoculated into animals these strains were classified as non-STEC (Table1).

\begin{tabular}{ccc}
\hline & Group III & Group IV \\
\hline Weeks without inoculation & & \\
\hline 1 & 0.0 & 0.0 \\
2 & 0.0 & 0.0 \\
3 & 0.0 & 0.0 \\
Weeks post-inoculation & & \\
1 & $34 / 134$ & 0.0 \\
2 & $122 / 152$ & 0.0 \\
3 & $133 / 143$ & 0.0 \\
4 & $288 / 119$ & 0.0 \\
5 & $323 / 123$ & 0.0 \\
6 & $129 / 143$ & 0.0 \\
7 & $84 / 138$ & 0.0 \\
\hline
\end{tabular}

Table 1. Proportion of means of STEC with the means of ordinary E. coli grown on plate re-isolated from feces samples from sheep from Groups I to IV during three weeks without inoculation and then during seven weeks post-inoculation.

Ordinary strain of $E$. coli were all strains that not displayed similar DNA to the strains previously inoculated into animals

The relations among the means values of STEC non-O157 strains displaying the specific pattern of DNA previously inoculated with E. coli strains non-STEC from group II and III were respectively as follows: 21/123, 130/142, 146/135, 304/122, 352/132, 190/145 and 90/148; 
$34 / 134,122 / 152,133 / 143,288 / 119,323 / 123,129 / 143$ and 84/138 bacteria isolated per gram of feces. (Table1). The means values of STEC non-O157 strains displaying specific pattern of DNA previously inoculated in the animals from groups II and III were compared among itself within the same week to verify the possible reduction of isolates occurred in the animals from group III by administration of probiotics strains (Figure1).

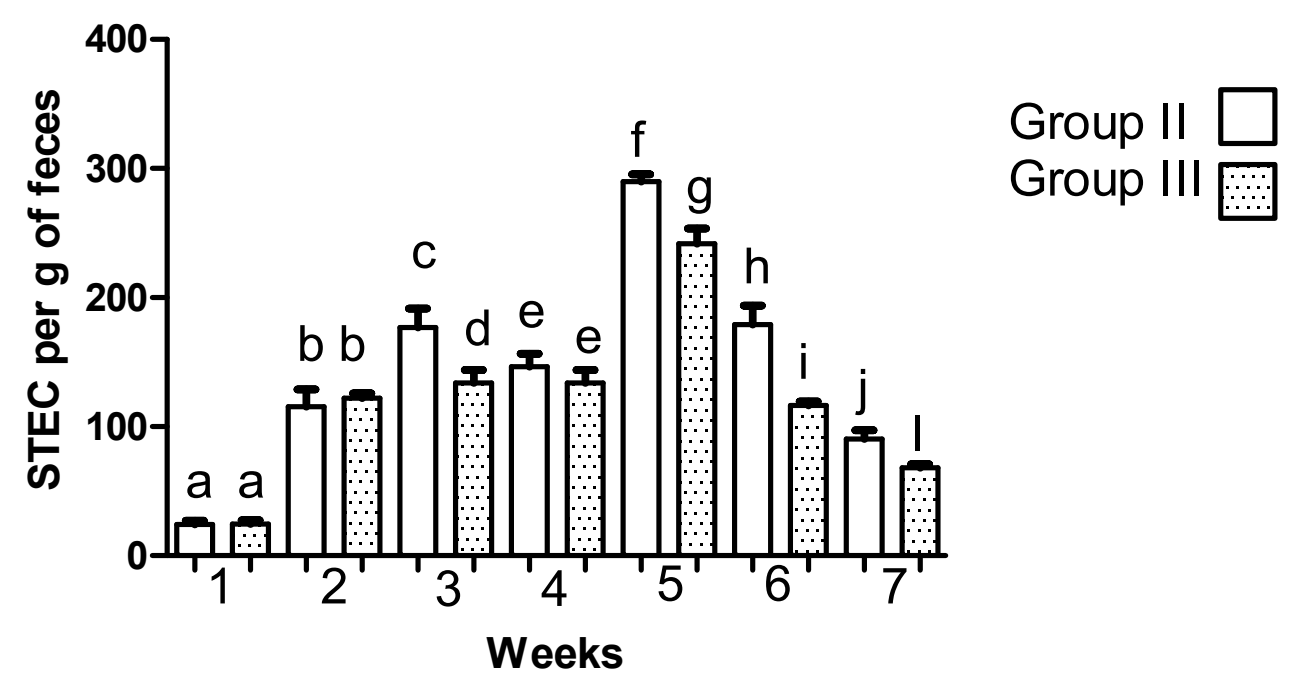

Figure 1. Comparison among the means of STEC from samples feces from Groups II and III. In each week the same letters show that the means not differs among them.

Comparing the means values of isolates of STEC non-157 strains from group II with the means values of isolates of STEC non-O157 strains from Group III within the same weeks verified that the difference was statistically significant among them only the third, fifth, sixth and seventh week post animals' inoculation (Figure1). There was lowest shedding of STEC non-O157 displaying similar DNA to the pattern of STEC non-O157 previously inoculated into animals belonged to the Group III than Group II, except in the first, second, and fourth week. The Group III had been received probiotic together with the STEC non-O157.

When the quantification was made through the selection at least five colonies from fecal sample during seven weeks of 1 to 5 sheep the results were 24, 26, 29, 30 and 29 in the group II and 20, 15, 19, 18, 16 in the group III (Table.2 and Table.3). The results show that there was no isolating of STEC non-O157 from sheep before the inoculation of bacteria inoculated. The total number of isolates from animals from group III were lowest than from group II. However these values not differ statistically. The aim this second counting was to verify if the reduction of shedding of STEC non-O157 from group III compared with group II would be shown by other way. However, this last counting way did not show statistical difference among the isolates. 


\begin{tabular}{cccccc}
\hline & Sheep1 & Sheep2 & Sheep3 & Sheep4 & Sheep5 \\
\hline Weeks post-inoculation & 2 & & & & \\
1 & 2 & 3 & 3 & 3 & 2 \\
2 & 5 & 4 & 5 & 5 & 4 \\
3 & 5 & 5 & 5 & 5 & 5 \\
4 & 4 & 5 & 4 & 5 & 5 \\
5 & 3 & 4 & 3 & 4 & 4 \\
6 & 3 & 3 & 4 & 4 & 4 \\
7 & $\mathbf{2 4}$ & $\mathbf{2 6}$ & $\mathbf{2 9}$ & $\mathbf{3 0}$ & $\mathbf{2 9}$ \\
\hline
\end{tabular}

Table 2. Total values of STEC re-isolated from feces sample selecting at least five colonies grown per samples from Group II.

\begin{tabular}{cccccc}
\hline & Sheep1 & Sheep2 & Sheep3 & Sheep4 & Sheep5 \\
\hline Weeks post-inoculation & 3 & & & & \\
1 & 3 & 2 & 3 & 3 & 2 \\
2 & 4 & 1 & 3 & 2 & 2 \\
3 & 3 & 3 & 2 & 3 & 2 \\
4 & 1 & 4 & 3 & 3 & 3 \\
5 & 4 & 2 & 3 & 2 & 3 \\
6 & 2 & 1 & 2 & 3 & 1 \\
7 & $\mathbf{2 0}$ & $\mathbf{1 5}$ & $\mathbf{1 9}$ & $\mathbf{1 8}$ & $\mathbf{1 6}$ \\
\hline
\end{tabular}

Table 3. Total values of STEC re-isolated from feces sample selecting at least five colonies grown per samples from Group III.

\section{Discussion}

Shiga-toxin-producing E. coli (STEC) strains are associated as a foodborne pathogen since 1982 and it has been identified as the cause of several outbreaks (Beutin et al., 2002; Karmali et al., 1989; Willshaw et al., 2001).

Probiotics are live microorganisms taken as food supplements that beneficially affect the host, maintaining a balance in their intestinal microbiota (Fuller, 1989). The ruminants including cattle, sheep and deer are reservoirs of STEC and the fecal shedding of these bacteria forms the vehicle of entry into the human food chain (Lema et al., 2001). The probiotics could be used as strategies to reduction of shedding these pathogens by animals (Chaucheyras-Durand et al. 2010).

In the present study we evaluated the protective effect of a mixture of probiotics strains to decrease the shedding of STEC non-O157 in sheep. The group III that received probiotic had fewer STEC non-O157 recovered from their feces when compared with the group II that did not receive the probiotics being that these differences were significant in $3^{\text {th }}, 5^{\text {th }}, 6^{\text {th }}$ to $7^{\text {th }}$ weeks. The probiotics strains failed to decrease the shedding of STEC non-O157 by feces 
during the first, second and fourth week post inoculation. In last three weeks of experiment there was a reduction in the shedding of the STEC non-O157 from feces from group III that received probiotic together with STEC non-O157 compared with the shedding of the STEC non-O157 from feces from group II which received STEC non-O157 only. For unknown reason the shedding of STEC non-O157 from group III was lower than group II during the third week post inoculation. However in the fourth week post inoculation there was no difference among the number of isolates of STEC non-O157 from both group III and II. As the probiotics beneficially affect the host, maintaining a balance in their intestinal microbiota (Fuller, 1989) probably the presence of probiotics strains hindered colonization and consequently the shedding these bacteria by feces.

Several mechanisms have been proposed to explain the beneficial effects of probiotics among them are the production of organic acids by bacterial probiotics can help decrease the gut $\mathrm{pH}$, create more favorable ecological conditions for the resident microbiota and decrease the risk of pathogen colonization (Servin, 2004). The growth of pathogenic bacteria also can be hindered by synthesis of antimicrobial peptides, such as bacteriocins or production of enzymes able to hydrolyze bacterial toxins (Buts, 2004), stimulating the immune system, increasing the absorption of minerals and increasing the syntheses of vitamins (Thuory et al., 2003). Bactericins are produced by many lactic acid bacteria (LAB), including species normally found in the gastrointestinal tract as L. acidophilus-group as $L$. acidophilus, Lactobacillus amylovorus, L. crispatus, L. crispatus, Lactobacillus gallinarum, L. gasseri and L. plantarum, (De Vuyst et al., 1996 and Dicks \& Botes, 2010).

Chaucheyras-Durand et al. (2010) indicated that some strategies may be used in the rumen to decrease the number of viable STEC cells as the use of Lactobacillus acidophilus supplemented in the ration, thereby preventing the contamination of food. These strategies are the administration of probiotics in the ruminants. The impact of probiotics and the physicochemical conditions of the rumen digesta on the survival of pathogenic strains could have significant implications for farm management practices and food safety and decrease the risk of food-borne illness.

In our study all sheep belonging to the group that received STEC non-O157 together with daily intake from probiotics strains had lower shedding this STEC non-O157. Some authors as Lema et al., (2001) verified that in lambs, the use of feed supplemented with lactic bacteria such as Lactobacillus acidophilus and Enterococcus faecium improved meat production. The mixture of probiotic strains used in this study contained strains of lactic bacteria, which probably allowed for the effect cited. Kritas et al., (2006) used Bacillus licheniformis and Bacillus subtilis supplemented in ration on sheep and verified although the mortality of sheep had not decreased there were beneficial effect on milk yields, fat and protein in milk.

As many bacterial species are present in the intestine, and under normal conditions the majority of these bacteria are strictly anaerobic. This composition makes the gut capable of responding to the possible anatomic and physicochemical variations that occur (Lee et al., 1999). The intestinal microbiota exercises a large influence on many biochemical reactions of the host. The balance maintained by probiotics hinders the growth of pathogenic 
microorganisms that are present. In contrast, an imbalance in the gut microbiota may cause the proliferation of pathogens and subsequent bacterial infection (Gibson, 1998).

The increased resistance against pathogens is the most important characteristic in developing effective probiotics. The use of probiotics strains excludes potentially pathogenic microorganisms and increases the natural defense mechanisms of the host (PuupponenPimiä et al., 2002). The modulation of intestinal microbiota by probiotic microorganisms occurs through a mechanism of competitive exclusion (Guarner and Malagelada, 2003). Also, the probiotics help to reset the intestinal microbiota through adhesion and colonization of the intestinal mucosa. This action hinders the adhesion or invasion of epithelial cells by pathogenic bacteria and decreases the synthesis of toxin. An imbalanced microbiota causes changes, such as the diarrhea associated with infections or treatment with antibiotics, allergic reactions to foods, and intestinal inflammatory diseases. Therefore, correcting an imbalance in the intestinal microbiota constitutes the basis for probiotic therapy (Isolauri et al., 2004). According Zhao et al. (1998), probiotics administered prior to exposure to pathogenic E. coli may reduce the levels of pathogenic E. coli carried in most animals. In this study we observed that concurrent inoculation of probiotics strains with STEC strains probably hindered the colonization of the pathogenic bacteria in the sheep, as compared with the groups that did not receive the probiotics treatment as well as by consequence decreasing thus the shedding by STEC non-O157. According to Batista et al. (2008), the administration of Lactobacillus acidophilus, decreased the number of days the animals displayed symptoms of diarrhea in the group of ruminants that received the probiotic compared with the group that did not receive any probiotic. Roos et al., (2010) verify that the use of Bacillus cereus and Sacharomyces boulardii enhanced the humoral immune response of lambs to the vaccines.

Some characteristics in probiotics strains are unwanted and much worrisome as well as antimicrobial resistance. Some lactic bacteria could present antibiotic resistance and these bacteria used for food is considered a major danger since this resistance could be transferred to pathogenic bacteria. The probiotics strains used in our study were tested to susceptibility to 27 antibiotics and verified that generally the Lactobacillus strains were inhibited to all antibiotics tested (Karapetkov et al., 2011).

In a study with cattle performed in Brazil, the authors used a probiotic contained strains of Ruminobacter amylophilus, Ruminobacter succinogenes, Succinovibrio dextrinosolvens, Bacillus cereus, Lactobacillus acidophilus and Streptococcus faecium, and these strains were administered at a dose of $3 \times 10^{8}$ live cells (CFU) of each strain resuspended in $250 \mathrm{~mL}$ of milk and administered orally. This study had many groups of animals. Some animals were vaccinated, others received probiotic and others both were vaccinated and received probiotic. These results showed that the combination of vaccine with the probiotic administered for 15 or 30 days were the most effective treatments for the control of diarrhea and weight gain (Ávila et al., 2000).

Some studies have indicated a higher prevalence of STEC in sheep than in cattle (Beutin et al., 1997; Sidjalat and Bensink, 1997; Urdahl et al., 2003), confirming that sheep are a 
significant reservoir of STEC. The findings of this study suggest that this probiotic likely presented a potential protective effect in reducing colonization by STEC non-O157 and can be used as an alternative method to decrease STEC non-157 infection in sheep, thereby reducing transmission to humans. Probiotic microorganisms, which benefit from a "natural image", can expect a promising future in animal nutrition (Chaucheyras-Durand and Durand, 2010).

\section{Author details}

Everlon Cid Rigobelo and Fernando Antonio de Ávila UNESP Animal Science Faculty of Dracena, UNESP Department of Veterinary Pathology, Brazil

\section{Acknowledgement}

The authors would like to thank FAPESP by financial support that permitted the realization of study. Process: 2009/14923-8

\section{References}

[1] Ávila, F., A., Paulillo, A., C. Schocken-Iturrino, R., P. et al., 2000. Evaluation of efficiency of a probiotic in the controlo $f$ diarrhea and weight gain in calves. Arquivos Brasileiros de Medicina Veterinaria e Zootecnia 41-46.

[2] Batista, C., G., Coelho, S., G., Rabelo, E., et al., 2008. Performance and health of calves fed milk without antimicrobials residue or milk from mastitis treated cows with or without probiotic. Arquivos. Brasileiro de Medicina Veterinária e Zootecnia. 185-191.

[3] Belongia, E.A., Osterholm, N.T., Soler, J.T., et al., 1993 Transmission of Escherichia coli O157: H7 infection in Minnesota child day-care facilities. Journal American. Medicine. Association. 269, pp. 883-888.

[4] Beutin, L., Geier, D., Zimmermann, S., et al., 1997. Epidemiological relatedness and clonal types of natural populations of Escherichia coli strains producing Shiga toxins in separate populations of cattle and sheep. Applied Environmental Microbiology 63, 2175-2180.

[5] Beutin, L., Kaulfuss, S., Cheasty, T., Brandenburg, B. Zimmermann, S., Gleier, K., Willshaw, G.A., Smith, H.R., 2002. Characteristics and association with disease of two major subclones of Shiga toxin (Verocytotoxin)-producing strains of Escherichia coli (STEC) O157 that are present among isolates from patients in Germany. Diagnostic Microbiology Infect Diseases, 44, 337-346.

[6] Buts, J.P., 2004. Exemple dún medicament probiotique: Sacchamoryces boulardii lyophilize. In Rambaud, J.C., Buts, J.P., Corthier, G. and Flourié, B. (eds) Flore microbienne intestinale. John Libbey Eurotext, Montrouge, France, pp.221-244.

[7] Chaucheyras-Durand, F., Fahima, F., Ameilbonne, A., et al., 2010. Fates of acid-resistant and non-acid-resistant shiga toxin-producing Escherichia coli strains in rumiant digestive 
contents in the absence and presence of probiotics. Applied. Environmental. Microbiology, 640-647.

[8] Chaucheyras-Durant, F., Durant, H., 2010. Probiotics in animal nutrition and health. Beneficial Microbes, 1, 3-9.

[9] China B, Pirson V, and Mainil J. 1996. Typing of bovine attaching and effacing Escherichia coli bymultiplex amplification of virulence-associated genes. Applied. Environmental. Microbiology. 82:3462-3463.

[10] Cross., M., 2002. Microbes versus microbes: immune signals generated by probiotic lactobacilli and their role in protection against microbial pathogens. FEMS, 245-253.

[11] De Vuyst, L., Callewart, R. and Pot, B., 1996. Characterization of the antagonistic activity of Lactobacillus amylovorus DCE 471 and large scale isolation of its bacteriocin amylovorin L471. Systematic and Applied Microbiology 19: 9-20.

[12] Dicks, 1.M.T., Botes, M. Probiotic lactic acid bacteria in the gastro-intestinal tract: health benefits, safety and mode of action. 2010. Beneficial Microbes, 1, 11-29.

[13] Food And Agriculture Organization Of The United Nations, World Health Organization. Evaluation of health and nutritional properties of probiotics in food including powder milk with live lactic acid bacteria. Córdoba, 2003. 34p. Available at: http://ftp.fao.org/es/esn/food/ probioreport_en.pdf>. Accessed 03 Fev 2005.

[14] Fuller, R., 1989. Probiotics in man and animals. Journal Applied Bacteriology Oxford, 66, 365-378.

[15] Gibson, G., R., Roberfroid, M., B., 1998. Dietary modulation of the human colonic microbiota: introducing the concept of prebiotics. Journal Nutrition, 125, 1401-1412.

[16] Guarner, F., Malagelada, J., R., 2003. Gut flora in health and disease. Lancet, London, $360,512-518$.

[17] Hussein, H. S. 2007. Prevalence and pathogenicity of Shiga toxin producing Escherichia coli in beef cattle and their products. Journal of Animal Science, 85, E63-E73.

[18] Isolauri, E., Salminen, S., Ouwehand, A., C., 2004. Probiotics. Best Pract. Research. Clinical of Gastroenterology, 2, 299-313.

[19] Karapetkov, N., Geogieva, Rumyan, N., Karaivanova, E. 2011. Antibiotic susceptibility of different lactic acid bacteria strains, Beneficial Microbes, 2, 335-339.

[20] Karmali, M.A., 1989. Infection by verocytotoxin-producing Escherichia coli. Clinical Microbiology. Review, 2, 15-38.

[21] Kritas, S.K., Govaris, A., Christodoulopopoulos, G., Burriel, A.R. 2006. Effect of Bacillus licheniformis and Bacillus subtilis supplementation of Ewe's feed on sheep milk production and young lamb mortality. Journal of Veterinary Medicine,53, 170-173.

[22] Lee, Y., K., Nomoto, K., Salminen, S., Gorbach, S., L., 1999. Handbook of probiotics. New York, Wiley, 211pp.

[23] Lema, M., Williams, L., Rao, D.R., 2001. Reduction of fecal shedding of enterohemorrhagic Escherichia coli O157:H7 in lambs by feeding by microbial feed supplement. Small Ruminant Research 39, 31-39.

[24] Posse B., Zutter, L.D., Heyndrickx, M., Herman, L. 2007. Metabolic and genetic profiling of clinical O157 and non-O157 Shiga-toxin-producing Escherichia coli, Institut Pasteur, $158,591-599$. 
[25] Puupponen-Pimiä, R., Aura, A., M., Oksmancaldentey, K., M., et al., 2002. Development of functional ingredients for gut health. Trends Food Science Technological, Amsterdam, 13, 3-11.

[26] Ramamurthy, T. 2008. Shiga toxin-producing Escherichia coli (STEC): the bug in our backyard. Indian Journal of Medical Research, 128, 233-236.

[27] Roos, T.B., Tabeleao, V.C., Dummer, L.A., et al., 2010. Effect of Bacillus cereus var Toyoi and Saccharomyces boulardii on the immune response of sheep to vaccines. Food and Agricultural Immunology, 21,113-118.

[28] Sanders, M., E., Klaenhammer, T., R., 2003. Invited review: the scientific basis of Lactobacillus acidophilus NCFM functionality as a probiotic. Journal. Dairy Science, 84, 319-331.

[29] Servin, A.I., 2004. Antagonistic activities of lactobacilli and bifidobacteria against microbial pathogens. FEMS Microbiology Reviews 28:405-440.

[30] Sidjabat, H., Bensink, J., C., 1997. Verotoxin-producing Escherichia coli from the faeces of sheep, calves and pigs. Australian Veterinary Journal 75, 292-293.

[31] Thuory, K., M., Probert, H., M., Smejkal, C., W., et al., 2003. Using probiotics and prebiotics to improve gut health. Drug Discovery Today, Haywards Heath,15, 692-700.

[32] Urdahl, A., M., Beutin, L., Skjerve, E., Zimmermann, S., et al., 2003. Animal host associated differences in Shiga toxin-producing Escherichia coli isolated from sheep and cattle on the same farm. Journal Applied Microbiology. 92-101.

[33] Wani S.,A, Bhat M., A, Samanta I, Nishikawa Y, and Buchh A., S. 2003. Isolation and characterization of Shiga toxinproducing Escherichia coli (STEC) and enteropathogenic Escherichia coli (EPEC) from calves and lambs with diarrhea in India. Letters. Applied. Microbiology. 37: 121-126.

[34] Willshaw, G.A., Cheasty, T., Smith, H.R., O'Brien, S.J., Adak, G.K., 2001. Verocytotoxinproducing Escherichia coli (VTEC) O157 and other VTEC from human infections in England and Wales: 1995-1998. Journal Medicine Microbiology, 50, 135-142.

[35] Zhao, T., Doyle, M., Harmon, B., et al., 1998. Reduction of carriage of enterohemorrhagic Escherichia coli O157:H7 in cattle by inoculation with probiotic bacteria. Journal Clinical Microbiology, 641-647. 